\title{
Brain metabolism and spatial memory are affected by portal hypertension
}

\author{
Natalia Arias • Marta Méndez • Jaime Arias • \\ Jorge L. Arias
}

Received: 18 October 2011 / Accepted: 19 January 2012 / Published online: 8 February 2012

(C) Springer Science+Business Media, LLC 2012

\begin{abstract}
Portal hypertension is a major complication of cirrhosis that frequently leads to a neuropsychiatric disorder that affects cognition. The present study was undertaken in order to compare the performance of sham-operated rats (SHAM) and portal hypertension rats $(\mathrm{PH})$ in reference memory tasks in the Morris water maze (MWM). Two groups of animals were used: SHAM group $(n=12)$ was used as a control group and PH group $(n=12)$ by the triple portal vein ligation method was used as an animal model of early evolutive phase of PH. The portal pressure was measured in the splenic parenchyma. Our work shows that spatial learning in the MWM is not impaired in PH group although this group showed a one-day delay in the task acquisition compared to the SHAM group. We assessed the brain metabolic activity of the animals by means of cytochrome c-oxidase (COx) histochemistry. Significant changes were found in the CA3, dentate gyrus, basolateral, medial, lateral and central amygdala, showing lower COx activity in the $\mathrm{PH}$ group as compared to the SHAM group in all cases. We found no changes in metabolic activity in prefrontal cortex and CA1 area between groups. In fact, different neural networks were shown according to the execution level of the subjects. The early PH evolution
\end{abstract}

N. Arias $\cdot$ M. Méndez $\cdot$ J. L. Arias $(\bowtie)$

Laboratorio de Neurociencias, Departamento de Psicología,

Universidad de Oviedo,

Plaza Feijoo s/n,

CP: 33003 Oviedo, Asturias, Spain

e-mail: jarias@uniovi.es

J. Arias

Departamento de Cirugía I, Facultad de Medicina,

Universidad Complutense de Madrid,

Ciudad Universitaria $\mathrm{s} / \mathrm{n}$,

28040 Madrid, Spain induced changes in brain metabolic activity without biggest alterations in spatial memory.

Keywords Acute portal hypertension - Memory networks · Cytochrome c-oxidase $\cdot$ Limbic system $\cdot$ Rat

\section{Introduction}

Hypertension is the single most important risk factor for cardiovascular disease and, thus, remains a global public health challenge (Zubcevic et al. 2011). It has been extremely difficult to manage hypertension in $\approx 40 \%$ of hypertensive patients (Primatesta et al. 2001). Studies showed that systemic oxidative stress, inflammation and priming of peripheral polymorphonuclear leukocytes antecede the development of hypertension in experimental model of hypertension in the Sabra rat (Sela et al. 2004).

In humans portal hypertension is characterized by prolonged elevation of portal venous pressure greater than $10 \mathrm{mmHg}$ (Al-Ghamdi 2011). It is estimated that for every $1 \mathrm{mmHg}$ increase in hepatic venous pressure gradient above a threshold level of $10 \mathrm{mmHg}$, an $11 \%$ rise in the risk of clinical decompensation could be expected (Al-Ghamdi 2011). This growth in portal pressure results from both an increase in resistance to portal flow and an increment in portal blood flow (Carey 2011). Portal hypertension (PH) is a common complication of cirrhosis which is in association with distinctive risks, including luminal gut bleeding, ascites and hepatic encephalopathy (HE) (Carey 2011).

There are diverse experimental models that have been developed to study the pathogenic mechanism that may be responsible for HE symptoms (Butterworth et al. 2009). Type B, which concerns HE related to portosystemic shunt and does not require any hepatocytic alteration, includes 
portocaval shunt, partial ligature of the portal vein and triple portal vein ligation (Orloff 2001).

Alterations in learning and memory capacity related to spatial memory and object recognition in rats with $\mathrm{PH}$ have been previously described in adult animals and in 18-monthold rats (Méndez et al. 2009a; Begega et al. 2010). The brain structures related to this kind of memory are the prefrontal cortex (Ragozzino et al. 1999) and the hippocampus, mainly the rostral part, which is involved in the memory for spatial information (Moser et al. 1995).

The objective of this work was to evaluate motor changes and to assess learning impairment at early evolutive phases of PH. For this purpose, we assessed the impact of PH by triple portal vein ligation on spatial reference memory and we studied oxidative metabolism of different brain limbic system regions involved in memory process by histochemical labelling of cytochrome oxidase (COx). COx is a mitochondrial enzyme involved in the phosphorylation process that generates ATP. This energy is used to maintain the resting membrane potential and the synthesis of molecules and neurotransmitters, among other functions (GonzálezLima and Cada 1994). Because metabolic activity is tightly coupled with neuronal activity, this technique can be used as an index of regional functional activity in the brain, reflecting changes in tissue metabolic capacity induced by sustained energy requirements of the nervous system associated with learning (Poremba et al. 1998) and spatial memory in the Morris Water Maze (MWM) (Conejo et al. 2007; de la Torre et al. 1997).

\section{Material and Methods}

\section{Subject}

A total of 24 male Wistar rats were used (3 months at the start of the experiment) from the animalarium of Oviedo University. All the animals had ad libitum food and tap water and were maintained at constant room temperature $\left(22 \pm 2^{\circ} \mathrm{C}\right)$, with a relative humidity of $65 \pm 5^{\circ} \mathrm{C}$ and artificial light-dark cycle of $12 \mathrm{~h}(08: 00-20: 00 / 20: 00-08: 00)$. The procedures and manipulation of the animals used in this study were carried out according to the Directive (2010/ 63/EU) and Royal Decree 1201/2005 of the Ministry of Presidency relating to the protection of the animals used for experimentation and other scientific purposes, and the study was approved by the local committee for animal studies (Oviedo University).

Procurement of experimental models

The animals were randomly distributed into two groups: portal hypertension (PH group, $n=12$ ) and sham-operated
(SHAM group, $n=12$ ). The experimental models that require surgery were anesthetized by i.m. injection of ketamine $(100 \mathrm{mg} / \mathrm{kg})$ and xylacine $(12 \mathrm{mg} / \mathrm{kg})$. With regard to postsurgical care, rats were kept near a heat source $(10$ $15 \mathrm{~min}$ ) until they regained consciousness to prevent postoperative hypothermia. They were then introduced into individual polycarbonate cages for 15 days and subsequently grouped into cages of four animals until their behavioral evaluation.

\section{Portal hypertension}

A midline abdominal incision was performed and a section of the intestinal loops was gently shifted to the left and covered with saline-moistened gauze. The portal vein was isolated and three ligatures, fixed on a sylactic guide, were performed in its superior, middle and inferior portions. The stenoses were calibrated by simultaneous ligation (4-0 silk) around the portal vein and a 20 -gauge blunt-tipped needle. The abdominal incision was closed in two layers with catgut and 2-0 silk. The postoperative period started immediately after the intervention and lasted until the behavioral evaluation 45 days later.

\section{Sham-operated}

A bilateral subcostal laparotomy with prolongation to the xyphoid apophysis, followed by isolation of the portal vein, was performed. The operative field was irrigated with saline solution during the intervention as the portal hypertension ones. Finally, the laparotomics were closed by continuous suture on the two layers with catgut and $2-0$ silk. The postoperative period started immediately after the intervention and lasted until the behavioral evaluation 45 days later.

\section{Portal vein pressure measurement}

Splenic pulp pressure, an indirect measurement of portal pressure (PP), was measured by the method described by Aller et al. (2006).

Determination of neurological alterations and motor functions

\section{Neurological test}

Before intervention and after the postoperative period, all the animals were handled during $10 \mathrm{~min}$ and evaluated by neurological tests such as flexion reflex, startle reactions, head shacking, righting and equilibrium reflex, placing reactions, grasping and some equilibrium tests. No alteration was displayed by any animal. 


\section{Rotarod-accelerod test}

This test of motor performance consists of a motor-driven rotating rod that enables us to assess motor coordination and resistance to fatigue (Jones and Roberts 1968). The accelerating rotarod 7750 of Ugo Basile (Ugo Basile Biological Research Apparatus, Italy) was used for the rats. The procedure followed has two parts. In the first one, the animals were placed in the apparatus and the speed was maintained constant at $2 \mathrm{rpm}$ for $60 \mathrm{~s}$. In the second part, the rats were evaluated for $5 \mathrm{~min}$ in the accelerod test session, in which the rotation rate constantly increased until it reached $20 \mathrm{rpm}$. Latency to fall off the rod and the actual rotation speed were recorded.

\section{Evaluation of spatial reference memory}

Spatial reference memory was evaluated in the circular pool designed by Morris (Morris 1984) also called the Morris water maze (MWM), one of the experimental paradigms most widely used to evaluate spatial memory. The MWM was made of fibreglass and measured $150 \mathrm{~cm}$ in diameter with a wall $40 \mathrm{~cm}$ high. The water level was $30 \mathrm{~cm}$ and its temperature $22 \pm 2^{\circ} \mathrm{C}$. The platform used corresponded to a cylinder $10 \mathrm{~cm}$ in diameter and $28 \mathrm{~cm}$ high, of which $2 \mathrm{~cm}$ was below the surface of the water. The MWM was in the centre of a $16 \mathrm{~m}^{2}$ lit room (two halogen lamps of $4000 \mathrm{~lx}$ ) and was surrounded by panels on which the spatial clues were placed. The pool was divided into four imaginary quadrants (quadrant $\mathrm{A}, \mathrm{B}, \mathrm{C}$ and $\mathrm{D}$, or right, left, across and correct, respectively). The behaviour of the animal in the MWM was recorded by a video camera (Sony V88E) connected to a computer equipped with an EthoVision Pro programme.

The learning protocol consisted of the first day being destined for habituation of animals to the task, in which the animals carried out four trials with a visible platform that jutted out $4 \mathrm{~cm}$ from the water and was located in the centre of the pool. During the following 5 days, the animals were required to locate a hidden platform that remained in the centre of quadrant $\mathrm{D}$ in relation to the external visual cues on training days. Training was performed in blocks of six trials per day. To begin each trial, the rats were placed in the water, facing the maze wall, at one of four quadrants, and the daily order of entry into these quadrants was randomized. Each trial ended once the animal had found the hidden platform or when $60 \mathrm{~s}$ had elapsed. If the animal had not reached the hidden platform after this time, it was placed on the platform for $15 \mathrm{~s}$. During the inter-trial interval, the animals were placed in a black bucket for $30 \mathrm{~s}$. The time and distance swum in each trial were recorded. At the end of the session, a probe test was applied in which the pool platform was removed and the rat was introduced into the pool in the quadrant opposite to where the platform had been located in previous trials for $25 \mathrm{~s}$ in order to check whether the animal remembers the position of the platform (Méndez et al. 2010). Immediately after the probe test, the animals were subjected to an additional trial with the hidden platform placed in its usual position to avoid any possible interference with the probe test. Latencies were recorded during the acquisition and also the time of permanence in each quadrant during the probe test.

\section{Cytochrome oxidase histochemistry}

Ninety min after the last training day animals were decapitated. Brains were removed, frozen rapidly in N-methylbutane (Sigma-Aldrich, Madrid, Spain) and stored at $-40^{\circ} \mathrm{C}$ until processing with quantitative $\mathrm{COx}$ histochemistry, described by González-Lima and Cada (1994). To quantify enzymatic activity and to control staining variability across different baths, sets of tissue homogenate standards from Wistar rat brain were cut at different thicknesses $(10,30,40$ and $60 \mu \mathrm{m})$ and included with each bath of slides. The sections and standards were incubated for $5 \mathrm{~min}$ in 0.1 phosphate buffer with $10 \%(\mathrm{w} / \mathrm{v})$ sucrose and $0.5(\mathrm{v} / \mathrm{v})$ glutaraldehyde, $\mathrm{pH}$ 7.6. After this, baths of $0.1 \mathrm{M}$ phosphate buffer with sucrose were given for $5 \mathrm{~min}$ each. Subsequently, $0.05 \mathrm{M}$ Tris buffer, $\mathrm{pH}$ 7.6, with $275 \mathrm{mg} / \mathrm{l}$ cobalt chloride, sucrose, and 0.5 (v/v) dimethylsulfoxide was applied for $10 \mathrm{~min}$. Then, sections and standards were incubated in a solution of $0.06 \mathrm{~g}$ cytochrome c, $0.016 \mathrm{~g}$ catalase, $40 \mathrm{~g}$ sucrose, $2 \mathrm{ml}$ dimethylsulfoxide and $0.4 \mathrm{~g}$ diaminobenzidine tetrahydrochloride (Sigma-Aldrich, Madrid, Spain), in $800 \mathrm{ml}$ of $0.1 \mathrm{M}$ phosphate buffer, at $37^{\circ} \mathrm{C}$ for $1 \mathrm{~h}$. The reaction was stopped by fixing the tissue in buffered $4 \%(\mathrm{v} / \mathrm{v})$ formalin for $30 \mathrm{~min}$ at room temperature. Finally the slides were dehydrated, cleared with xylene and coverslipped with Entellan (Merck, Germany).

Quantification of COx histochemical staining intensity was done by densitometric analysis, using a computerassisted image analysis workstation (MCID, Interfocus Imaging Ltd., Linton, England) made up of a high precision illuminator, a digital camera and a computer with specific image analysis software. The mean optical density (OD) of each region was measured on bilateral structures, using three consecutive sections in each subject. In each section, four non-overlapping readings were taken, using a squareshaped sampling window that was adjusted for each region size. A total of twelve measurements were taken per region by an investigator blind to the groups. These measurements were averaged to obtain one mean per region for each animal. OD values were then converted to COx activity units, determined by the enzymatic activity of the standards measured spectrophotometrically (González-Lima and Cada 1994). 
The regions of interest were anatomically defined according to Paxinos and Watson's atlas (2005) (Paxinos and Watson 2005). The regions of interest and the distance in $\mathrm{mm}$ of the regions counted from bregma was: $+3.20 \mathrm{~mm}$ for the infralimbic cortex (IL), prelimbic cortex (PL), the cingulate cortex (CG); $-1.20 \mathrm{~mm}$ for the CA1, CA3 and the dentate gyrus (DG) subfields of the dorsal hippocampus; $-3.12 \mathrm{~mm}$ for the basolateral (Bas), medial (MeA), lateral (Lat) and central (Cen) amygdala.

\section{Statistical analysis}

\section{Behavioral data}

Motor data were analyzed by t-test for independent samples. For the velocity and distance covered, we used a two way repeated measures ANOVA (between factor: group; within factor: day, five levels) as well as to analyse the latencies to reach the hidden platform for each day (average of four trials) during the training phase of the reference memory task. The time spent in each of the four quadrants during the probe test was analysed separately for each group and day, using a one way ANOVA design (factor: quadrants, four levels). Post hoc multiple comparisons analysis was carried out, when allowed, using the Holm-Sidak method. Moreover, a non-parametric Mann-Whitney $U$ test $(\mathrm{H})$ for independent samples was carried out when normality or equal group variances failed.

\section{COx activity}

Group differences in COx activity measured in each brain region were evaluated by Student's t-test for independent samples. Because training experience in spatial learning might manifest as neural changes in functional connectivity, the possible functional relationships among the regional brain activity data were analysed in terms of pairwise correlations within each experimental group. For the interregional correlation analysis, Pearson's product-moment correlations between pairs of brain regions in each experimental group were computed. COx activity values were normalized by dividing the measured activity of each brain region by the average COx activity value for all regions measured in each animal. This was done to reduce variation in the intensity of the COx staining not resulting from the experimental manipulation. In addition, in order to avoid errors due to an excessive number of significant correlations using small sample sizes, a "jackknife" procedure was used (Shao and Dongsheng 1995). This procedure is based on the calculation of all possible pairwise correlations resulting from removing one subject each time, and taking into consideration only those correlations that remain significant $(p<0,05)$ across all possible combinations.

\section{Results}

Portal vein pressure measurement

Portal pressure increases in rats with $\mathrm{PH}$ which showed mean values of $14.844 \pm 2.08$ respect to the SHAM group where portal pressure values were $5.903 \pm 0.43$.

Rotarod-accelered test

A t-test for independent samples revealed no significant differences between groups in the rotarod/accelerod. The PH group did not differ from the SHAM group in the rpm reached by the rotarod $(\mathrm{t}(22)=1.243, p=0.227)$ or in time in seconds the animals managed to withstand at fast speed $(\mathrm{t}(22)=1.294, p=0.209)$ (Fig. 1).

\section{Reference memory}

The two-way repeated measures ANOVA revealed no differences in distance covered $(\mathrm{F}(1,88)=2.490, p=0.129)$ and

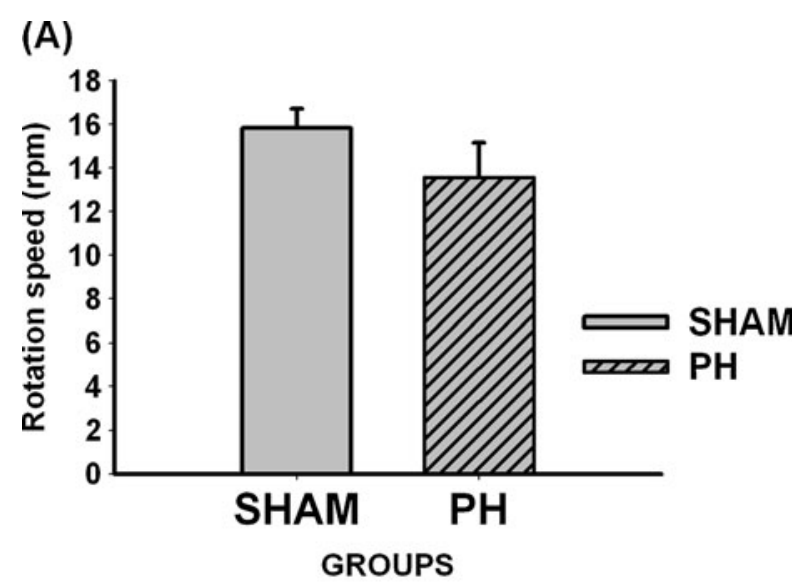

(B)

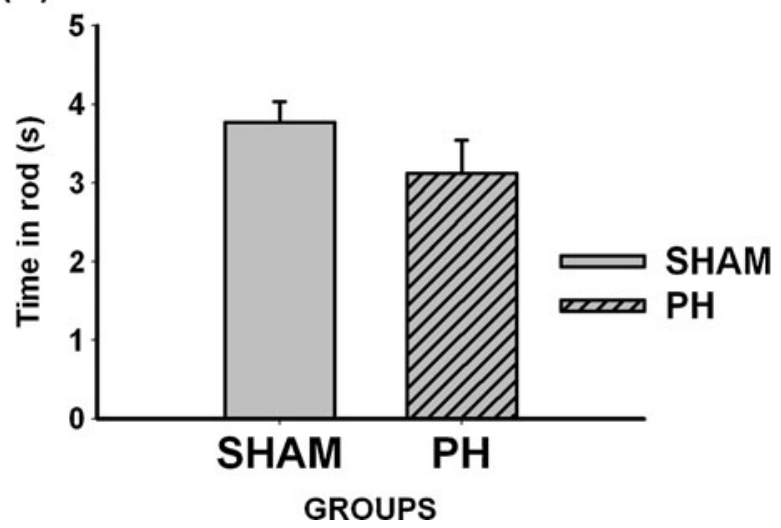

Fig. 1 Rotarod test. Bars charts represent rotation speed a and time spent on the rod $\mathbf{b}$ in the rotarod/accelerod test. There were no statistically significant differences between the groups (mean \pm SEM) 
velocity $(\mathrm{F}(1,88)=1.321, p=0.263)$ between $\mathrm{PH}$ and SHAM. The variable day, however, did have a significant effect $(\mathrm{F}(4,88)=26.460, p<0.001)$ and $(\mathrm{F}(4,88)=4.040$, $p=0.005$ ), respectively (Fig. 2).

The two-way repeated measures ANOVA showed no differences in escape latencies between the PH and SHAM groups $(\mathrm{F}(1,88)=0.462, p=0.504)$. However, significant differences until day three $(p=0.006)$ in the escape latencies to reach the hidden platform were found in SHAM group respect to the $\mathrm{PH}$ group which showed a one-day delay in the task acquisition ( $p=0.007)$ (Fig. 3).

Analysis of the time spent in the target quadrant during the probe tests showed an improvement in learning over the 5 days of training for all groups. But, whereas the SHAM group acquired the task in only a few trials, only from Day 3 did permanence in the reinforced quadrant prevail (Day 1: $\mathrm{F}$ $(3,44)=4.323, p=0.009$; Day 2: $\mathrm{H}(3)=10.122, p=0.018$; Day 3: $\mathrm{F}(3,44)=23.764, p<0.001$; Day 4: $\mathrm{H}(3)=26.423$, $p<0.001$; Day 5: $\mathrm{H}(3)=26.716, p<0.001)$. Post hoc tests revealed significant differences between Quadrant $\mathrm{D}$ and the rest of quadrants $(p \leq 0.005)$ on these days. However, the $\mathrm{PH}$ group showed a predominant permanence in Quadrant $\mathrm{D}$ on

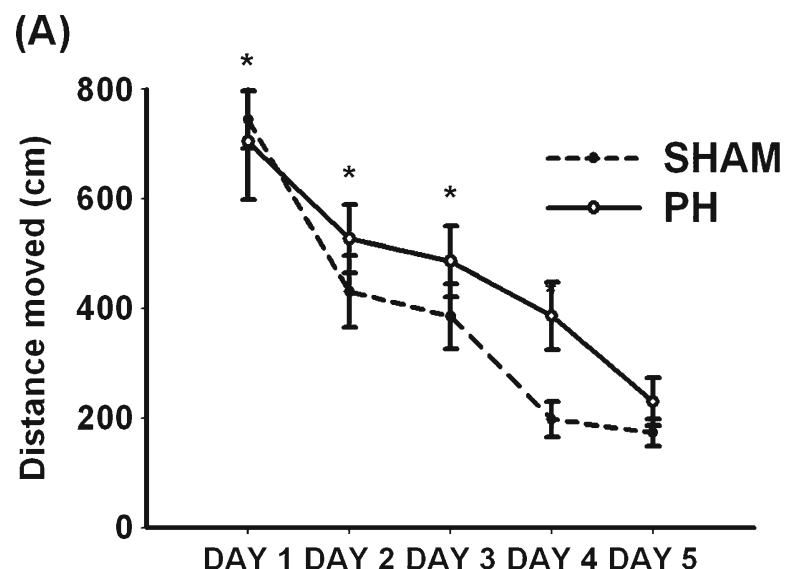

(B)

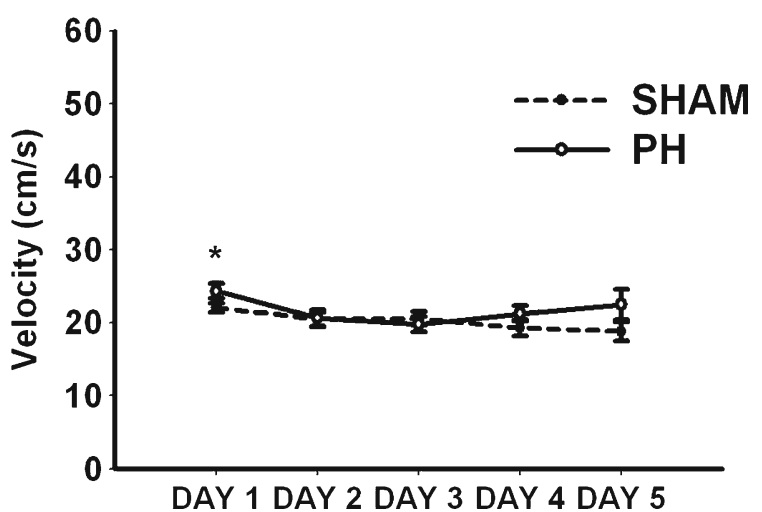

Fig. 2 Distance moved and Velocity. Bars charts (mean \pm SEM) represent distance moved a and velocity $\mathbf{b}$. There was a significant effect of the variable day $(* p \leq 0.05)$
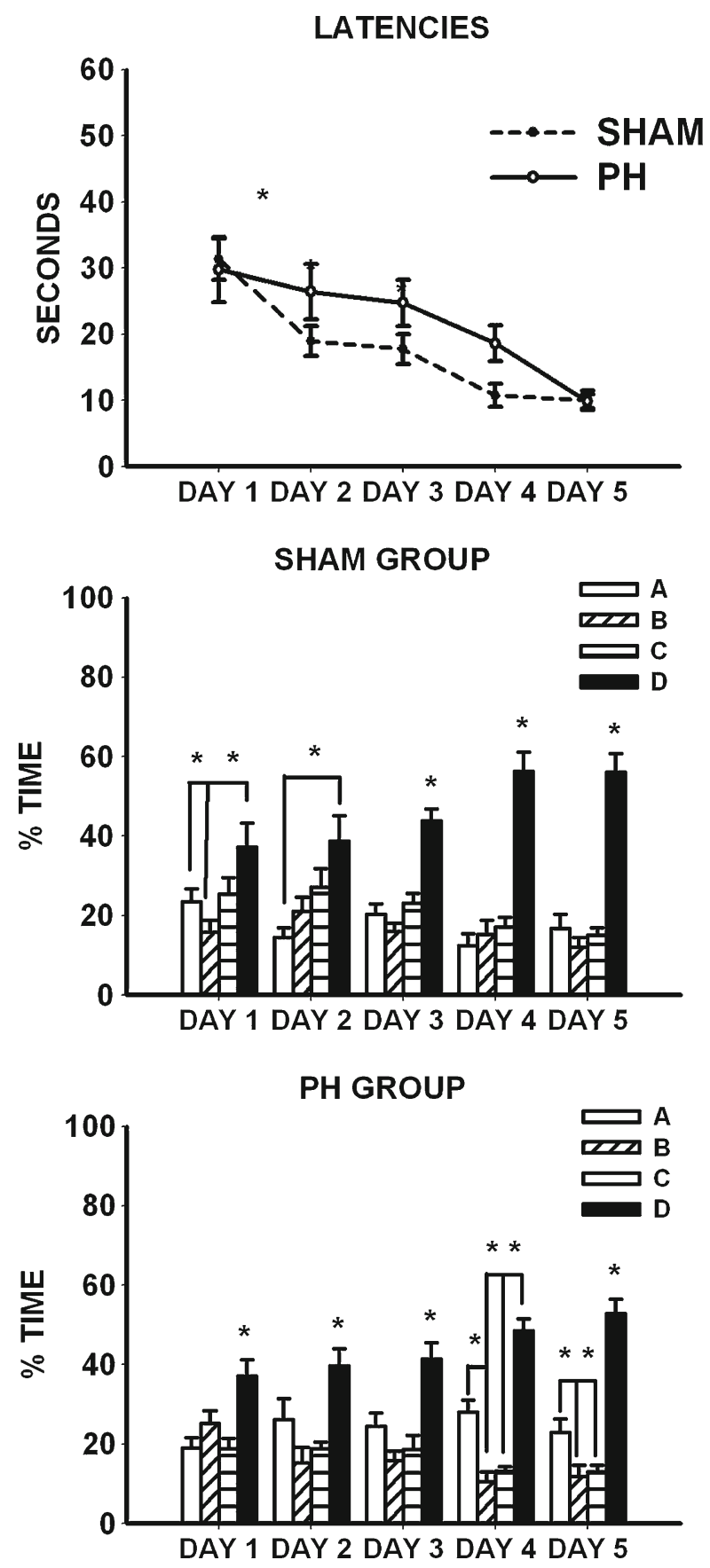

Fig. 3 Escape latencies and probe tests in the spatial referencememory test during 5 days learning (mean $\pm \mathrm{SEM}$ ). The escape platform was located in quadrant $\mathrm{D}$. There was an improvement over days for all groups in escape latencies $(* p \leq 0.05)$. $\mathrm{PH}$ group showed one-day delay in the correct execution of the transfer test respect to the SHAM group

the three first days (Day 1: $\mathrm{F}(3,44)=7.231, p<0.001$; Day $2: \mathrm{F}$ $(3,44)=7.024, p<0.001$; Day 3: $\mathrm{F}(3,44)=11.368, p<0.001)$ and significant differences between the other quadrants were found on Days 4 and 5 (Day 4: $\mathrm{H}(3)=33.565, p<0.001$; Day 5: $\mathrm{F}(3,44)=41.253, p<0.001)$. Post hoc tests for Day 4 revealed significant differences $(\mathrm{p} \leq 0.005)$ between Quadrants D-B, D-C and A-B. But Day 5 showed differences 
between Quadrant D and the rest of quadrants $(p<$ $0.001)$ and between Quadrants A-B $(p=0.010)$ and Quadrant A compared to Quadrant C $(p=0.022)$ (Fig. 3).

\section{COx activity}

The group comparison of COx activity revealed lower COx activity in the $\mathrm{PH}$ group than in the SHAM group in the CA3 $(U=166.000, \mathrm{n} 1=11, \mathrm{n} 2=12 ; p=0.039)$ and $\mathrm{DG}$ $(U=171.000, \mathrm{n} 1=11, \mathrm{n} 2=12 ; p=0.018)$, Bas $(U=165.000$, $\mathrm{n} 1=11, \mathrm{n} 2=12 ; p=0.045)$, Lat $(U=165.000, \mathrm{n} 1=11, \mathrm{n} 2=12$; $p=0.045)$, Cen $(U=168.000, \mathrm{n} 1=11, \mathrm{n} 2=12 ; p=0.029)$, and $\operatorname{MeA}(U=173.000, \mathrm{n} 1=11, \mathrm{n} 2=12 ; p=0.013)$. The groups did not differ in their COx activity in the PL $(U=148.000$, $\mathrm{n} 1=11, \mathrm{n} 2=12 ; p=0.340), \mathrm{IL}(U=156.000, \mathrm{n} 1=11, \mathrm{n} 2=12$; $p=0,148)$, and $\mathrm{CG}(U=153.000, \mathrm{n} 1=11, \mathrm{n} 2=12 ; p=0.207)$ (Table 1).

\section{Interregional within-group correlations of $\mathrm{COx}$ activity}

Significant interregional correlations of COx activity were found both in the SHAM and the PH groups. A high crosscorrelation between medial amygdala and CA1 subfield ( $r=$ $-0.853, p<0.001)$, between CA1 subfield and CA3 subfield $(r=0.903, p<0.001)$ as well as between CA3 subfield and central amygdala $(r=-0.794, p=0.004)$ were found in the SHAM group. In contrast, interregional correlations in the $\mathrm{PH}$ group were found between the medial and lateral amygdala $(r=0.766, p=0.004)$, the lateral amygdala and CA1 subfield $(r=-0.685, p=0.014)$, the CA1 subfield and CA3 subfield ( $r=0.720, p=0.008)$, as well as between the prelimbic cortex and infralimbic cortex $(r=0.735, p=0.006)$, the infralimbic cortex and central amygdala $(r=-0.693, p=0.012)$, the central and cingulate cortex $(r=-0.817, p=0.001)$ (Fig. 4).

Table 1 Shows the COx values (mean \pm SEM) in SHAM and PH groups for all structures studied

\begin{tabular}{lcc}
\hline Structures & SHAM group & PH group \\
\hline Cornu ammonis 3 & $21,74 \pm 1,88$ & $16,32 \pm 0,49$ \\
Dentate gyrus & $33,45 \pm 3,11$ & $23,41 \pm 0,50$ \\
Basolateral amygdala & $29,68 \pm 2,79$ & $20,82 \pm 0,38$ \\
Lateral amygdala & $22,25 \pm 1,90$ & $15,64 \pm 0,39$ \\
Central amygdala & $24,19 \pm 1,92$ & $17,58 \pm 0,53$ \\
Medial amygdala & $22,31 \pm 1,57$ & $16,62 \pm 0,37$ \\
Prelimbic cortex & $22,49 \pm 1,75$ & $18,36 \pm 0,46$ \\
Infralimbic cortex & $23,11 \pm 1,73$ & $18,47 \pm 0,50$ \\
Cingulate cortex & $22,99 \pm 1,83$ & $18,20 \pm 0,49$ \\
\hline
\end{tabular}

\section{Discussion}

Our work shows that spatial learning in the MWM is delayed in rats with acute prehepatic $\mathrm{PH}$, also significant changes could be observed in the COx activity between groups. These differences cannot be justified by motor problems, as indicated in the tests carried out specifically for this purpose.

Patients with cirrhosis show a neuropsychiatric disorder called minimal hepatic encephalopathy (MHE) which, while clinically (neurologically) free of symptoms, manifest abnormalities in central nervous system function where assesed using psychometric tests or electrophysiological techniques. Significant impairment of selective attention and executive function has been reported in MHE, together with abnormalities in prolongation of cognitive evoked potentials, visuomotor activity, response inhibition, slowing of the electroencephalogram and psychomotor speed (Amodio et al. 2004). These deficits have been associated with alterations in the functional connections within the basal ganglia-thalamo-cortical loop (Timmermann et al. 2002; Timmermann et al. 2003). In contrast, in the experimental model of PH employed our results from the rotarodaccelerod test revealed that these alterations were not reflected. Our groups presented a balanced motor coordination and similar displacement speed and distance, in agreement with others authors (Méndez et al. 2008). Indeed, it can be explained by the different neuronal circuits which are activated in normal rats and in rats with HE to modulate motor activity (Cauli et al. 2009). This modulation is due to the sequential alterations in glutamatergic (Canales et al. 2003) and GABAergic neurotransmission in different brain areas involved in these circuits.

Although SHAM and PH groups reached the behavioral criterion, the $\mathrm{PH}$ group tended to perform worse than the SHAM group in the reference memory task. In fact, there was a one-day delay in the reduction in the escape latencies to a hidden platform during the learning process for the $\mathrm{PH}$ group. Analysis of the probe tests, in which the platform was removed from the pool, revealed that, although there were no group differences in the time of permanence in the target quadrant, the SHAM group increased the time of permanence in the reinforced quadrant during the last 3 days of the acquisition tasks, whereas the $\mathrm{PH}$ group showed significant differences between the other quadrants on the last 2 days evaluated. These results are in agreement with those obtained by Méndez and cols (2008) in the study of the working memory. Working memory is a representation of an object, stimulus or spatial location that is typically used within a testing session (Dudchenko 2004), which requires a degree of cognitive flexibility and short-term storage. The data of this study could indicate that the $\mathrm{PH}$ group presented more deficits in short-term memory than in long-term spatial memory. In fact, several studies with 
Fig. 4 Schematic diagram showing the significant interregional correlations of COx activity calculated in the studies groups (SHAM and $\mathrm{PH})$. Solid and dotted lines represent respectively highly positive and negative pair-wise Pearson's correlations $(r>0.8$, $P<0.02)$
SHAM group

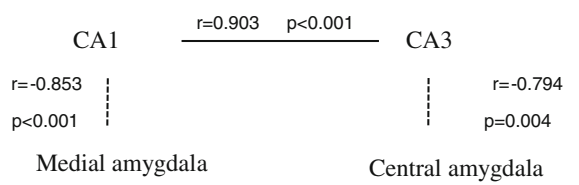

PH group

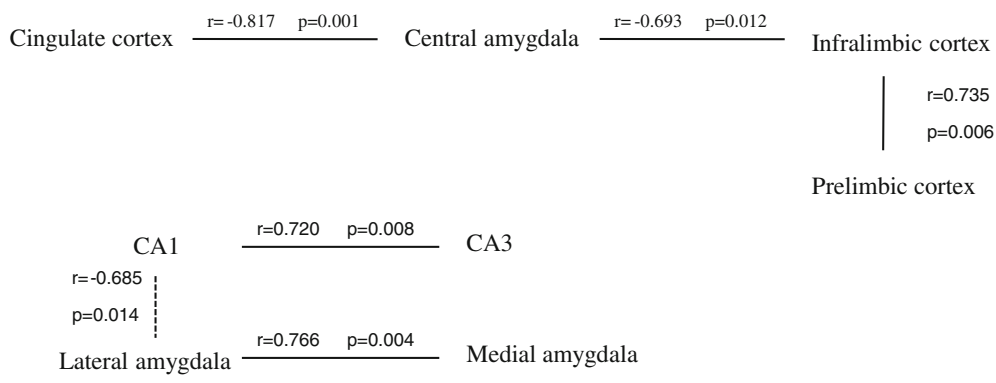

different experimental models of HE confirms that spatial working memory is damaged in cirrhotic rats due to the weekly administration of thioacetamide. This experimental group was unable to remember the location of platform in the retention trial, displaying difficulties in recalling new spatial information for a short period of time (Méndez et al. 2009a). Moreover, studies that enable us to evaluate the typical avoidance response, where an increase in the number of avoidances on the different acquisition days can be attributed to processes of consolidation and recall, showed that the portacaval shunt group and thioacetamide group had a clear deficit compared to control groups (Méndez et al. 2009b). All these data led to the hypothesis that massive exposition to the acquisition task during the 5 days on which the reference memory is carried out is probably the cause of the absence of differences between groups.

Histochemical labelling of COx revealed an absence of changes between groups in the mPFC area (PL, IL and CG) which is in agreement with the lack of motor dysfunctions, planning (prospective coding) and decision making (Kesner and Churchwell 2011). Dorsal regions of mPFC such as CG have been implicated in various motor behaviours, while ventral regions of $\mathrm{mPFC}$ (PL and IL) have been associated with diverse emotional, cognitive and mnemonic processes (Heidbreder and Groenewegen 2003).

The same pattern of activity could be observed in the CA1 subfield of the hippocampus. Previous studies have revealed projections from the hippocampus (CA1/subiculum) to the ventral mPFC (IL/PL) (Carr and Sesack 1996; Jay and Witter 1991), stronger to IL than to PL and to the dorsal mPFC (Hoover and Vertes 2007). It is known that hippocampal neurons encode newly-learnt goal locations through the reorganization of assembly firing patterns in the $\mathrm{CA} 1$ region but not in $\mathrm{CA} 3$. This suggests specialization within the hippocampus in order to solve spatial problems: whereas CA1 place representations are flexible because it encodes goal locations and the successful retrieval of goalassociated spatial memories, CA3 are stable, providing invariant representations of the whole environment independently of task demand (Dupret et al. 2010). In addition, Dupret and cols. (2011) have shown that enhanced reactivation of newly-established goal representations is NMDA receptor-dependent. These findings highlight the important role of NMDA receptor-dependent hippocampal plasticity during learning and are consistent with other behavioral studies showing that formation of spatial memories involves NMDA receptor (Nakazawa et al. 2004; McDonald et al. 2005).

Regarding the hippocampus, we observed that the dentate gyrus (DG) and CA3 subfield of the hippocampus were under-activated in the $\mathrm{PH}$ group. This could be related to the moderate spatial deficit showed in the task. Indeed, in experimental models where the advanced stages of the HE were studied, it has been found that an increase of the ammonia levels impairs induction of NMDA receptordependent long-term potentiation in the hippocampus (Méndez et al. 2010) and also alters the function of the glutamate-NO-cGMP pathway, resulting in reduced formation of cGMP (Montoliu et al. 2010). The changes in extracellular cGMP are parallel to changes in learning ability, supporting a role for cGMP levels in learning ability (Llansola et al. 2007). Moreover, behavioral studies suggest that spatial learning both of entire environments and discrete places involves NMDA receptor-dependent plasticity to update the hippocampal representation of space according to task demands and/or environmental changes. But these NMDA receptors are not only located in the hippocampus. Recent research has shown the importance of glutamate receptors in the basolateral complex of the amygdala (Bas) and in the central nucleus of the amygdala (Cen) (Llansola et al. 2007). 
Furthermore, significant reciprocal inhibitory connections have been described between the ventral prefrontal cortex and amygdala (Womer et al. 2009).

The neurobehavioral correlations identified different patterns of interactivity in networks of brain regions between groups. In the SHAM group, which showed more metabolic activity than the $\mathrm{PH}$ group, we could observe a simple circuit that involves limbic regions associated with the hippocampal formation (CA1 and CA3) and included in Papez' $\mathrm{s}$ circuit, such as the amygdala (Cen and MeA). Others studies revealed CA3-CA1 uncoupling in 5-day trained animals, and these regions correlated with behavioural learning scores in 3-day trained animals (Conejo et al. 2010). It is known that the learning process is reached near Day 3, so we hypothesized that this CA3-CA1 coupling indicates a delay in the brain activity in both groups, which is not reflected in the behavioural learning. In fact, other authors have highlighted that the performance of a laparotomy may cause some alterations that are still uncertain (López et al. 1997).

The PH group showed two more complex networks. The first one included relationships between CA1 and CA3 subfields of the hippocampus with other nuclei of amygdala such as MeA and Lat. The second network involved regions of the mPFC (CG, PL and IL) and Cen nuclei of the amygdala. This amount of structures could indicate metabolic brain efforts in the $\mathrm{PH}$ group, which are necessary to acquire the spatial reference memory in the same time as the SHAM group.

The PH rats showed no spatial learning impairment but there were differences in brain metabolic activity. This study is important because it revealed a metabolic deficit associated with some learning impairment at early phases of $\mathrm{PH}$. These results support the complicated diagnosis related to the MHE which are unavailable to most clinicians. In fact, MHE patients have increased risk of developing and a lower survival rate (Romero-Gómez et al. 2007). Thus, others studies could be aimed at early detection of these symptoms in humans in order to prevent the chronic stages of the illness.

Acknowledgements We would like to thank Piedad Burgos, Begoña Valdés for their technical assistance, Lucía Ibañez for her surgical contributions. This research was supported by grant from the Spanish Ministry of Science and Innovation (PSI2010-19348), FMMA (AP/ 6977-2009) and MEC AP2009-1714 to N. Arias.

\section{References}

Al-Ghamdi H (2011) Carvedilol in the treatment of portal hypertension. Saudi J Gastroenterol 17:155-158. doi:10.4103/13193767.77251
Aller MA, Vara E, García C, Nava MP, Angulo A, Sánchez-Patán F, Calderón A, Vergara P, Arias J (2006) Hepatic lipid metabolism changes in short- and long-term prehepatic portal hypertensive rats. World J Gastroenterol 12:6828-6834

Amodio P, Montagnese S, Gatta A, Morgan MY (2004) Characteristics of minimal hepatic encephalopathy. Metab Brain Dis 19:253-267. doi:10.1023/B:MEBR.0000043975.01841.de

Begega A, Méndez M, Rubio S, Santín LJ, Aller MA, Arias J, Arias JL (2010) (2010) Portal hypertension in 18-month-old rats: memory deficits and brain metabolic activity. Physiol Behav 100:135-142. doi:10.1016/j.physbeh.2010.02.014

Butterworth RF, Norenberg MD, Felipo V, Ferenci P, Albrecht J, Blei AT, Members of the ISHEN Commission on Experimental Models of HE (2009) Experimental models of hepatic encephalopathy: ISHEN guidelines. Liver Int 29:783-788. doi:10.1111/j.14783231.2009.02034.x

Canales JJ, Elayadi A, Errami M, Llansola M, Cauli O, Felipo V (2003) Chronic hyperammonemia alters motor and neurochemical responses to activation of group I metabotropic glutamate receptors in the nucleus accumbens in rats in vivo. Neurobiol Dis 14:380-90. doi:10.1016/j.nbd.2003.08.023

Carey W (2011) Portal hypertension: diagnosis and management with particular reference to variceal hemorrhage. J Dig Dis 12:25-32. doi:10.1111/j.1751-2980.2010.00473.x

Carr DB, Sesack SR (1996) Hippocampal afferents to the rat prefrontal cortex: synaptic targets and relation to dopamine terminals. J Comp Neurol 369:1-15

Cauli O, Rodrigo R, Llansola M, Montoliu C, Monfort P, Piedrafita B, El Mlili N, Boix J, Agustí A, Felipo V (2009) Glutamatergic and gabaergic neurotransmission and neuronal circuits in hepatic encephalopathy. Metab Brain Dis 24:69-80. doi:10.1007/s11011008-9115-4

Conejo NM, González-Pardo H, González-Lima F, Arias JL (2010) Spatial learning of the water maze: progression of brain circuits mapped with cytochrome oxidase histochemistry. Neurobiol Learn Mem 93:362-371. doi:10.1016/j. nlm.2009.12.002

Conejo NM, González-Pardo H, Vallejo G, Arias JL (2007) Changes in brain oxidative metabolism induced by water maze training. Neuroscience 145:403-412. doi:10.1016/j.neuroscience.2006.11.057

de la Torre JC, Cada A, Nelson N, Davis G, Sutherland RJ, GonzalezLima F (1997) Reduced cytochrome oxidase and memory dysfunction after chronic brain ischemia in aged rats. Neurosci Lett 223:165-168. doi:10.1016/S0304-3940(97)13421-8

Dudchenko PA (2004) An overview of the tasks used to test working memory in rodents. Neurosci Biobehav Rev 28:699-709. doi:10.1016/j.neubiorev.2004.09.002

Dupret D, O'Neill J, Pleydell-Bouverie B, Csicsvari J (2010) The reorganization and reactivation of hippocampal maps predict spatial memory performance. Nat Neurosci 13:995-1002. doi:10.1038/ nn. 2599

González-Lima F, Cada A (1994) Cytochrome oxidase activity in the auditory system of the mouse: a qualitative and quantitative histochemical study. Neuroscience 63:559-578. doi:10.1016/ 0306-4522(94)90550-9

Heidbreder CA, Groenewegen HJ (2003) The medial prefrontal cortex in the rat: evidence for a dorso-ventral distinction based upon functional and anatomical characteristics. Neurosci Biobehav Rev 27:555-579. doi:10.1016/j.neubiorev.2003.09.003

Hoover WB, Vertes RP (2007) Anatomical analysis of afferent projections to the medial prefrontal cortex in the rat. Brain Struct Funct 212:149-179. doi:10.1007/s00429-007-0150-4

Jay TM, Witter MP (1991) Distribution of hippocampal CA1 and subicular efferents in the prefrontal cortex of the rat studied by means of anterograde transport of Phaseolus vulgarisleucoagglutinin. J Comp Neurol 313:574-586 
Jones BJ, Roberts DJ (1968) The quantitative measurement of motor incoordination in naïve mice using an accelerating rotarod. J Pharm Pharmacol 20:302-304

Kesner RP, Churchwell JC (2011) An analysis of rat prefrontal cortex in mediating executive function. Neurobiol Learn Mem 96:417431. doi:10.1016/j.nlm.2011.07.002

Llansola M, Rodrigo R, Monfort P, Montoliu C, Kosenko E, Cauli O, Piedrafita B, El Mlili N, Felipo V (2007) NMDA receptors in hyperammonemia and hepatic encephalopathy. Metab Brain Dis 22:321-335. doi:10.1007/s11011-007-9067-0

López L, Burgos P, Santín LJ, Begega A, Arias J, Lorente L, Arias JL (1997) Portacaval shunt control animals: physiological consequences derived from the sham operation. Lab Anim 31:225230. doi: $10.1258 / 002367797780596239$

McDonald RJ, Hong NS, Craig LA, Holahan MR, Louis M, Muller RU (2005) NMDA-receptor blockade by CPP impairs posttraining consolidation of a rapidly acquired spatial representation in rat hippocampus. Eur J Neurosci 22:1201-1213. doi:10.1111/ j.1460-9568.2005.04272.x

Méndez M, Méndez-López M, López L, Aller MA, Arias J, Arias JL (2009a) Basal and learning task-related brain oxidative metabolism in cirrhotic rats. Brain Res Bull 78:195-201. doi:10.1016/j. brainresbull.2008.10.008

Méndez M, Méndez-López M, López L, Aller MA, Arias J, Arias JL (2009b) Associative learning deficit in two experimental models of hepatic encephalopathy. Behav Brain Res 198:346-351. doi:10.1016/j.bbr.2008.11.015

Méndez M, Méndez-López M, López L, Aller MA, Arias J, Cimadevilla JM, Arias JL (2008) Spatial memory alterations in three models of hepatic encephalopathy. Behav Brain Res 188:32-40. doi:10.1016/j.bbr.2007.10.019

Méndez M, Méndez-López M, López L, Begega A, Aller MA, Arias J, Arias JL (2010) Reversal learning impairment and alterations in the prefrontal cortex and the hippocampus in a model of portosystemic hepatic encephalopathy. Acta Neurol Belg 110:246-254

Montoliu C, Rodrigo R, Monfort P, Llansola M, Cauli O, Boix J, El Mlili N, Agusti A, Felipo V (2010) Cyclic GMP pathways in hepatic encephalopathy. Neurological and therapeutic implications. Metab Brain Dis 25:39-48. doi:10.1007/s11011-0109184-z

Morris RGM (1984) Developments of a water-maze procedure for studying spatial learning in the rat. J Neurosci Meth 11:47-60. doi:10.1016/0165-0270(84)90007-4
Moser MB, Moser EI, Forrest E, Andersen P, Morris RG (1995) Spatial learning with a minislab in the dorsal hippocampus. Proc Natl Acad Sci U S A 92:9697-96701

Nakazawa K, McHugh TJ, Wilson MA, Tonegawa S (2004) NMDA receptors, place cells and hippocampal spatial memory. Nat Rev Neurosci 5:361-372. doi:10.1038/nrn1385

Orloff MJ. (2001) Portal hypertension and Portocaval Shunt, Academic Press p. 637-701.

Paxinos G, Watson CH. (2005) The rat brain in stereotaxic coordinatesthe new coronal set. 5th ed. Elsevier Academic Press

Poremba A, Jones D, González-Lima F (1998) Classical conditioning modifies cytochrome oxidase activity in the auditory system. Eur J Neurosci 10:3035-3043. doi:10.1046/j.1460-9568.1998.00304.x

Primatesta P, Brookes M, Poulter NR (2001) Improved hypertension management and control: results from the health survey for England 1998. Hypertension 38:827-832

Ragozzino ME, Detrick S, Kesner RP (1999) Involvement of the prelimbic-infralimbic areas of the rodent prefrontal cortex in behavioral flexibility for place and response learning. J Neurosci 19:4585-4594

Romero-Gómez M, Córdoba J, Jover R, del Olmo JA, Ramírez M, Rey R, de Madaria E, Montoliu C, Nuñez D, Flavia M, Compañy L, Rodrigo JM, Felipo V (2007) Value of the critical flicker frequency in patients with minimal hepatic encephalopathy. Hepatology 45:879-885. doi:10.1002/hep.21586

Sela S, Mazor R, Amsalam M, Yagil C, Yagil Y, Kristal B (2004) Primed polymorphonuclear leukocytes, oxidative stress, and inflammation antecede hypertension in the Sabra rat. Hypertension 44:764-769. doi:10.1161/01.HYP.0000144480.10207.34

Shao J, Dongsheng T (1995) The jackknife and bootstrap. Springer, New York

Timmermann L, Gross J, Butz M, Kircheis G, Häussinger D, Schnitzler A (2003) Mini-asterixis in hepatic encephalopathy induced by pathologic thalamo-motor-cortical coupling. Neurology 61:689-692

Timmermann L, Gross J, Kircheis G, Häussinger D, Schnitzler A (2002) Cortical origin of mini-asterixis in hepatic encephalopathy. Neurology 58:295-298

Womer FY, Kalmar JH, Wang F, Blumberg HP (2009) A ventral prefrontal-amygdala neural system in bipolar disorder: A view from neuroimaging research. Acta Neuropsychiatr 21:228-238

Zubcevic J, Waki H, Raizada MK, Paton JF (2011) Autonomicimmune-vascular interaction: an emerging concept for neurogenic hypertension. Hypertension 57:1026-1033. doi:10.1161/ HYPERTENSIONAHA.111.169748 